\title{
Socially-Aware Device-To-Device Communications
}

\author{
Dhananjay Sinha1, T. Lakshmi Kiran'1, Dr. L. Mary Gladence² \\ 1UG Student, Department of CSE, Sathyabama Institute of Science and Technology, Chennai, India \\ ${ }^{2}$ Assistant Professor, School of Computing, Sathyabama Institute of Science and Technology, Chennai, India
}

\begin{abstract}
How to cite this paper: Dhananjay Sinha / T. Lakshmi Kiran / Dr. L. Mary Gladence "Socially-Aware Device-ToDevice Communications" Published in International Journal of Trend in Scientific Research and Development (ijtsrd), ISSN: 24566470, Volume-3 I Issue-3, April 2019, pp. 79-84. URL: http://www.ijtsrd.co $\mathrm{m} /$ papers/ijtsrd2157 7.pdf

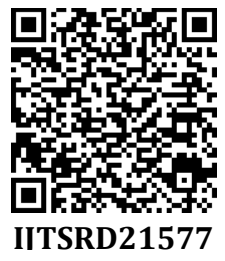

Copyright (c) 2019 by author(s) and International Journal of Trend in Scientific Research and Development Journal. This is an Open Access article distributed under the terms of the Creative Commons

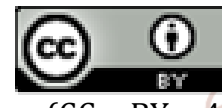
Attribution License (CC BY 4.0 ) (http://creativecommons.org/licenses/ by/4.0)

\section{INTRODUCTION}

Device to-Device (D2D) Communication [5] is a vital innovation for the up and coming age of versatile system foundations. It expects to expand the compelled capacities of a cell phone, to upgrade the Quality-of-Service (QoS) and Quality-of-Experience (QoE) of the end client. Basically, D2D is the crafty intersection of Devices that frames a fleeting and creative calculation framework, which can be utilized to encourage the consummation of an errand by dispersing the intricacy of its execution over different Devices. D2D misuses the entrepreneurial setting of the Devices, e.g., same area, a few correspondence channels, higher information data transfer capacity and speed, and so forth, so as to make an all the more dominant framework. The exertion of exe cuting an errand utilizing such framework is decreased when contrasted and the exertion required by a solitary Device to execute a similar undertaking. For example, a major record isolated into littler lumps can be transferred or downloaded quicker by various Devices instead of a solitary Device gushing the total document. What's more, a computational errand, e.g., QuickSort, NQueens, can be doled out to different Devices to perform load-adjusting, diminishing vitality utilization.

Normally, since the development of a D2D framework isn't constrained by an incorporated specialist, e.g., arrange administrator, but instead it relies upon the portability and enthusiasm of clients to rent their cell phones' assets, a D2D foundation depends on the egocentric conduct of the client.
Consequently, as of recently the majority of the work is center around estimating the potential applied advantages of D2D situations and researching components to cultivate client's investment. Socially-mindful worldview alludes to the finding of association designs among socially associated clients, and further using these examples to structure effective answers for the D2D correspondence arrange.

The social-setting includes data found from the disconnected, OSN and MSN profiles of clients (e.g., likes or remarks, share photographs, divider posts, and messages). This data significantly relates and relies upon the likeness among clients' interests (e.g., names in contact book and supporters in OSN), exercises (e.g., discourses and surveys) and their connections (e.g., family relationship and kinship). It ought not be blended with setting mindful that alludes to the capacity of a versatile clients' applications to find and react to changes in nature they have a place. These can be in client setting, physical setting or figuring setting [8]. The exploration endeavors on the intermingling of socialmindfulness with D2D interchanges are still in its early stages, and various difficulties should be managed to make it functional and extensively adequate.

D2D interchanges have gotten gigantic consideration from scientists since its initiation, and numerous studies have examined different parts of D2D correspondences [9]- [13]. On examination of the previously mentioned reviews, it 
substantiates the way that current best in class writing misses the mark concerning measuring sociality's effect on D2D interchanges. The consolidated D2D interchanges with social-space viewpoint covering the total specialized issues and the forthcoming applications have never been reviewed. To the best of creators' information, this is the principal complete overview on socially mindful D2D interchanges to connect this glaring hole. Theauthors in [9] secured a wide writing survey on a few specialized issues of D2D correspondences in LTE-A systems. In [10], grouping of D2D interchanges as for inband and outband D2D correspondences is displayed. While in [11], the creators proposed the design to satisfy the endorser requests, and talked about D2D correspondence key issues alongside its vital advancements. These fundamental innovations incorporate ultra-thick systems (UDNs), millimeter wave D2D (mmWave), and psychological D2D interchanges.

In [12], the social point of view is considered to take care of different issues for $5 \mathrm{G}$ systems joined with various system innovations. These incorporate web of things (IoT), web of vehicles (IoV), D2D correspondences, versatile distributed computing (MCC), keen lattices and huge information. In any case, socially empowered D2D correspondence is never been abridged and talked about. As of late, the creators in [13] just analyzed the ongoing advances in the area of sociallymindful asset portion and streamlining for D2D interchanges. Also, scientific classification was proposed dependent on the accompanying properties i.e., channeldriven, goals, unraveling approaches, organizing advancements, qualities, and correspondence types.

\section{RELATED WORK}

D2D correspondence is a viable nearness based worldview that empowers a client to appreciate the advantages of direct correspondence with high information rates and low deferrals. Be that as it may, before benefiting such a positive administration, it is expected to locate a reasonable friend inside the vicinity, which is a testing undertaking. There are a few issues inside this area that should be intelligently tended to. Trust is the premier issue that has pulled in the consideration of the educational network.

Zhang et al. [1] set forward a structure of socially mindful companion revelation that utilizes the social-connection data of network and centrality of CUs. In particular, the system is used to propose a specially appointed friend revelation plot that improves the information transmitting proportion. Further, the signal system is utilized to improve neighbor disclosure. Not at all like customary D2D plans that utilize arbitrary versatility models, the creators get the information from practical human portability, which gives increasingly sensible situations. In any case, CUs are not similarly liable to collaborate and have differing social associations with different clients inside a similar closeness. In this way, it is required to address this issue too. Close by, explore network considered numerous qualities for finding bunches dependent on clients' social-cooperations. The exact choice of such characteristics relies upon the sum of legitimate data accomplished, which can lead towards the better choice of neighboring D2D people group. In addition, covering gatherings can be found that can improve the transmission rates among between networks.
Wang et al. [3] progressed in the direction of this thought and proposed a system to distinguish the covering gatherings. All the more definitely, the creators concentrate such covering gatherings so as to recognize distinctive reference point discovery rates while breaking down unique jobs of clients. Afterward, these guide location rates are acclimated to diminish vitality utilization and to improve the neighboring disclosure rates. Be that as it may, the ideal signal identification rates are as yet not found to acquire the ideal D2D correspondence execution. In a resulting work, Tan et al. [3] proposed a model of companion disclosure and match component. Formally, the creators obtain the idea of Device's leftover vitality from green correspondence and afterward utilizing it as a coordinating procedure. Afterward, in light of these match results, a common asset assignment criteria is proposed for D2D interchanges. Further, all together to determine the demand strife to join a friend gathering, a fallback instrument is proposed with the goal that the quantity of D2D sets can be amplified. Another thought of companions is likewise presented in this paper brings up the distinction of physical and social relationship between two clients, yet at the same time they team up for augmenting the increases of D2D interchanges. So as to build up a connection between D2D sets, we need to ensure that the two Devices are situated in nearness.

Wang et al. [4] explored a confirmation approach by utilizing socialites, i.e., measurements among D2D clients, for example, contact recurrence and contact length for D2D interchanges. To figure the D2D connect strength and achievement rate before connection foundation, socialassociation data is fundamentally considered, in this manner, deliberately, they consider both the factual channel data and the measurable client versatility demonstrate

for D2D joins foundation. Their proposed strategies render a genuine guide for viable D2D interchanges underlay portable cell systems.

Socially-mindful D2D correspondence is required to change over the social-relationship among clients into helpful data for the basic leadership procedures of the comparing physical D2D systems. In such manner, Xiao et al. [5] proposed conviction based stable marriage amusement to break down socially-mindful D2D correspondences. This structure empowers every client to set up a conviction work with every other client. Moreover, the capacity is used further to detail a socially-mindful inclination for every single imaginable activity. Here, conviction work speaks to a likelihood dispersion over the potential social-associations with different clients. This can be open, private, static, and dynamic conviction capacities. Likewise, the creators researched range sharing issue for in-band D2D interchanges, and introduced how to use this system and advance the socially-mindful D2D correspondences.

Mao et al. [6] explored the collaboration throughput among different versatile hubs. The creators broke down the collaboration flag by means of an arbitrary variable, which epitomizes joint impact of both social and position connections to choose the participation likelihood and collaboration quality, separately. As participation throughput isn't the main capacity of the remote channel, along these lines, for throughput examination, the comparing capacity is determined, and the idea of summed up degrees of opportunity is used to research the framework execution conduct. Especially, an express plan between summed up 
degrees of opportunity, the social, and position connections is built up.

Mass et al. [10] set forward a customer server framework that uses the sound information of clients having socialbinds so as to naturally initiate D2D correspondences among them. All the more explicitly, the sound information is sent to the cloud to check whether the Devices share same closeness. Cloud performs machine learning examination over sound information as far as gathering the Devices by sound likeness. On the off chance that the Devices are recognized in a similar closeness, the cloud server tells the Devices to make a companion dependent on

the coordination plan. When the clients get such data from the cloud server, they manufacture an association with their gathering mates through Bluetooth asso ciations lastly share their documents. In spite of the fact that this proposition gives a low idleness structure to offload calculation, it isn't vitality effective as it needs to send information to the cloud in an occasional way.

Mao et al. [11] proposed an answer that recommends social and position based collaboration among clients to choose a confided in companion. In more detail, the creators presented a joint closeness metric that estimates the twolayered social position relationship in a bound together way. In the wake of figuring the measurement scores of conceivable companions, the values are arranged lastly the most appropriate friend is chosen for empowering D2D correspondences. Some way or another, this proposition settle the trust issue while consolidating social-relationship, however it expect that all the conceivable clients are agreeable, which is definitely not a sensible situation.

\section{D2D INFRASTRUCTURE SENSOR}

In haze and edge figuring settings, it is normal that a D2D framework will process a computational errand from different Devices in vicinity, e.g., sensors, shrewd watches, keen glasses, and so forth. While the exchange of a computational undertaking among Devices is a paltry errand in low inertness organizes, the arrangement of a D2D foundation is a mind boggling assignment as it requires, 1) the Devices to be discoverable among them 2) the foundation of stable intercommunication channels the coordination of the Devices so as to disseminate the preparing load.

Normally, a Device that isn't discoverable, it isn't addressable by others. Along these lines, it can't be misused for the formation of crafty foundation. A Device can be addressable by others by means of Bluetooth or WiFi-Direct advancements. Be that as it may, the addressability of a Device relies upon the versatile client, which is the one that stipends perceivability and consents to other people. In this work, we center around the discoverable issue, and left the rest as future work.

So as to gauge the amount D2D can add to mist and edge figuring conditions, we build up the Detector application, scans for accessible Devices by depending on Bluetooth and WiFi-Direct. The revelation of Devices happens every 15 minutes. We view this interim as enough so as to abstain from irritating the charging conduct of portable client. Naturally, Bluetooth and WiFi-Direct incites high vitality channel the Device. Along these lines, in the event that the disclosure procedure is trigger to frequently, at that point almost certainly, the client needs to charge his/her Devices at unexpected hours in comparison to the ordinary ones.

So as to gauge the amount D2D can add to mist and edge processing conditions, we build up the Detector application, scans for accessible Devices by depending on Bluetooth and WiFi-Direct. The revelation of Devices happens every 15 minutes. We view this interim as enough so as to abstain from irritating the charging conduct of portable client. As a matter of course, Bluetooth and WiFi-Direct actuates high vitality channel the Device. In this manner, on the off chance that the revelation procedure is trigger to frequently, at that point all things considered, the client needs to charge his/her Devices at unexpected hours in comparison to the customary ones.

\section{TECHNICAL CHALLENGES AND OPEN PROBLEMS}

\subsection{SECURITY AND PRIVACY}

Interpersonal organizations are advancing constantly and perceived with a huge number of rich data. This can reflect multifaceted social-associations among an immense number of clients with various individual interests and attributes. Besides, in any OSN, individual profile of clients may involve secret individual data combined with numerous other everyday exercises. In such manner, an inquiry emerges that how one can extricate basic and profitable social-data, without damaging clients' protection is as yet an open issue. OSN involves secret individual data. Hence, to guarantee mystery and protection, informal community administrators ought to set up secure interfaces between their system foundation and D2D correspondences to maintain a strategic distance from spillage of such data to unintended UEs.

\subsection{MOBILITY IMPACT}

Versatility factor is unimportantly considered in the sociallymindful D2D interchanges. For example, while examining the limit of impromptu systems, the hubs are viewed as fixed in greatest structures. The framework execution can be fundamentally improved by misusing client portability combined with social-attention to Devices. As we realize that information sending in D2D correspondence is incredibly subject to the way accessibility and versatility of the hubs. Thus, limit in such system increments because of hub versatility. Likewise, it is seen that delay in such systems is conversely relative to the versatile hub speed. Since individuals are social creatures, and thus, their versatility is for the most part affected by social perspectives and the network to which they have a place. Consequently, joining both the social-area and portability factor will open new roads, and help in better comprehension the down to earth D2D correspondence systems.

Displaying Practical and Efficient Approaches: There are different issues while considering traffic-offloading by means of D2D interchanges, yet introducing the substance dispersion by reasonable clients (seeds) choice is one of the imperative issues. The seeds must be amazingly potential ones to download the required substance documents and further to move around for sharing. Keeping this in view, some more difficulties should be tackled To know/evaluate the closeness of the substance, the creators utilized the technique to distinguish excess. In this methodology, a parcel level identification uses a reserve, and traffic payloads are 
fingerprinted by an improved rendition of the Jenkins hash. Information excess/comparability is identified through examination of the listed payloads of every parcel with the as of now stored information. A similar methodology can be utilized for evaluating comparable substance inside D2D systems with little changes.

Dispersed Efficient Schemes: It is incomprehensible for each UE to acquire the worldwide learning of interpersonal organization structure. An option is to depend on incorporated plans that require to securing worldwide data. It is lucky to embrace conveyed plan that enables every UE to take choices autonomously dependent on its own perceptions and data. At that point, it is appropriate to ask here that how to devise a proficient plan that can make accessible sensible motivating force for every UE to implement the required reasonableness in the basic leadership process. This is as yet an open issue.

\section{APPLICATIONS OF SOCIALLY-AWARE D2D COMMUNICATIONS}

In socially-mindful D2D correspondence arranges, every UE ought to think about the social-relations or associations with different UEs notwithstanding physical nearness and neighborhood asset accessibility. Social-mindfulness is viewed as a promising area for D2D correspondences as well as for other $5 \mathrm{G}$ innovations. It can give a further lift and improve the D2D interchanges in the accompanying angles i.e., storing and traffic offloading, computational offloading and substance spread. Hence, we classify socially-mindful D2D correspondence's applications into D2D storing and traffic offloading, computational offloading and substance dispersal

\section{EXISTING SYSTEM}

In existing idea individuals' straightforwardly went to the Upload side or call to the approved for the data. K-Means bunching plans to parcel $\mathrm{n}$ objects into k groups in which each article has a place with the group with the closest mean. This technique creates precisely k diverse bunches of most noteworthy conceivable qualification.

\subsection{DRAWBACKS EXISTING SYSTEM}

- It will take additional time.

\section{PROPOSED SYSTEM}

The proposed idea of an application is that the client can self-examine on social mindfulness on sifted society. Future improved development learning the issue to comprehend the arrangement on social mindfulness. The Apriori Algorithm is a compelling calculation for digging regular thing sets for Boolean affiliation rules. Apriori utilizes a "base up" approach, where visit subsets are broadened one thing at any given moment.

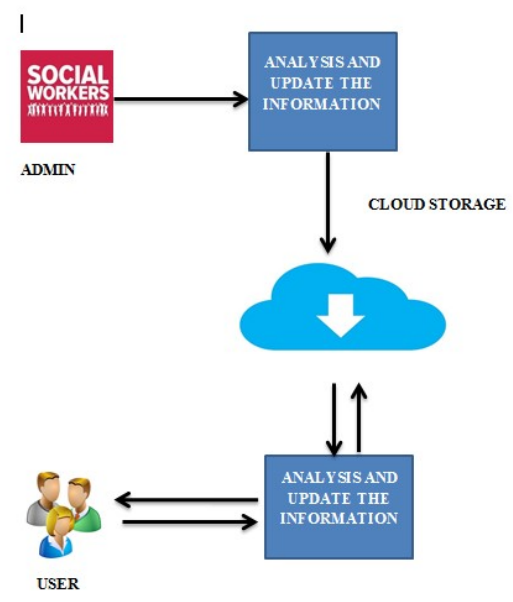

FIG 1 OVERVIEW OF PROPOSED SYSTEM

\subsection{ADVANTAGES PROPOSED SYSTEM}

- Straightforward and Time save. Has the quickest preparing on the cell phone.

\section{SYSTEM MODEL}

\section{$8.1 \quad$ LOGIN}

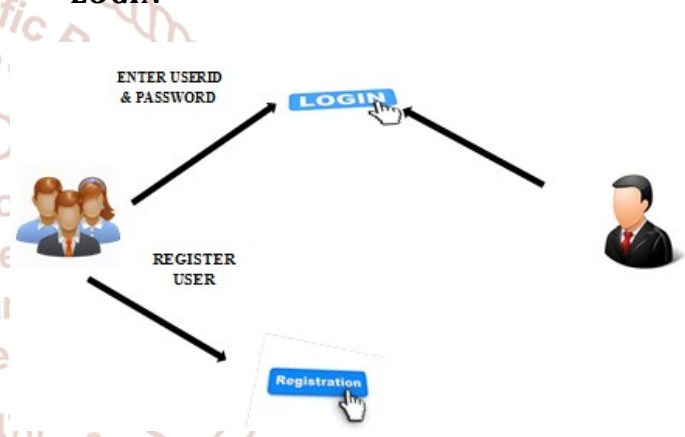

\section{FIG 2 LOGIN PROCESS}

In this module, we have plan for login and information exchange screen. Android utilized xml to create traditional screens in our application and java for coding. The information exchange page contains email id or client name, portable number, and secret key those sorts of subtleties ought to be put away in database. Login screen contains userid id or username and secret key.when the client, login the application it ought to recover the information to the database and join dependent on client input if its match client name, secret phrase to permit in the application else it gave an alarm and demonstrate a message to the client.

\subsection{DATABASE CREATION}

Client email id or client name and secret key have been put away after enrollment. Android utilized SQLite Database for putting away and bringing client application deta. 


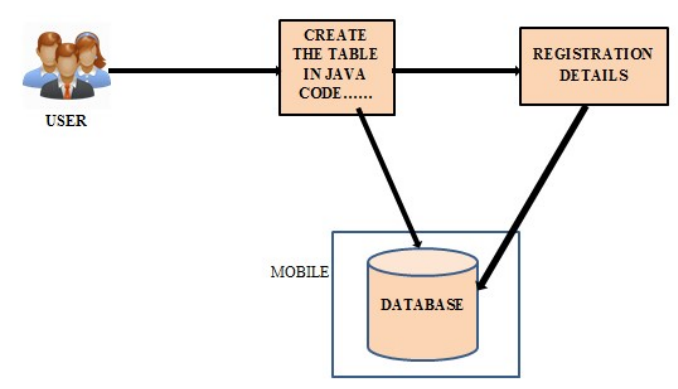

FIG 3 DATABASE CREATION

\subsection{UPLOAD INFORMATION}

In this module we intended for there is client enrollment structure accessible where new clients can make their record by giving expected data to the framework. Client can be will play out the transfer the data and administrator will see the information to screen the transferring procedure.

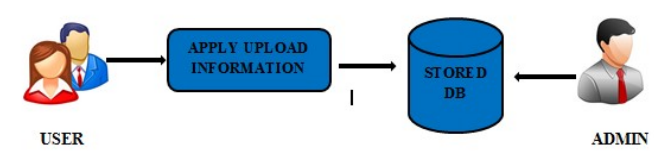

FIG 4 UPLOADS INFORMATION

\subsection{VIEW INFORMATION}

In this module, we have structure for view data are the client can be see the information of transferring data and administrator will check the procedure.

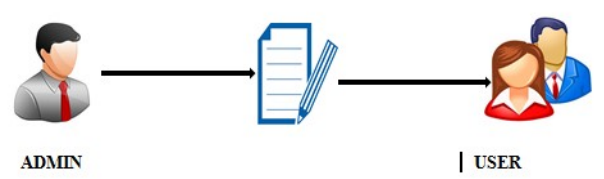

FIG 5 VIEW INFORMATION

\subsection{FEEDBACK VIEW UPLOAD REVIEW}

In this module, we are plan for input view transfer audit the client can be see the client transfer and view the remarks for criticism and view the criticism remarks.

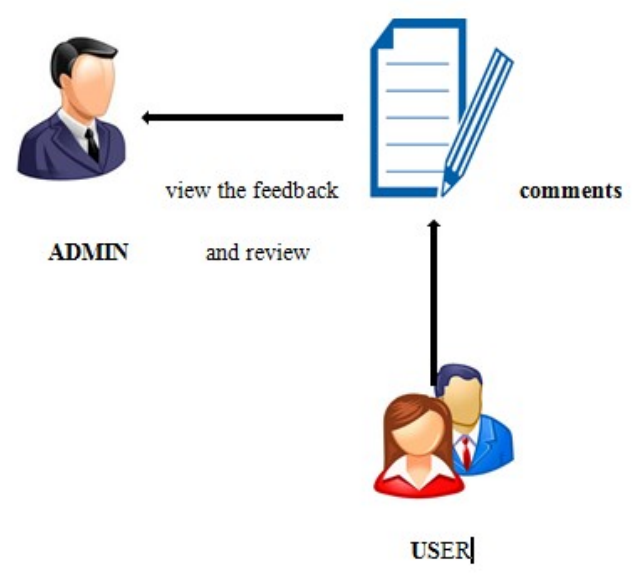

FIG 6 FEEDBACK VIEW UPLOAD REVIEW

\section{CONCLUSION}

In this paper, we plan Detector, a sensor to identify framework in nearness. We utilized our sensor to evaluate the measure of framework that is accessible in nature. Our examination gives bits of knowledge about the amount D2D correspondence can contribute towards the reception of Edge and Fog Computing. To make this conceivable, socialspace is utilized and combined with physical area to misuse the concealed advantages, and to adapt to the following correspondence age requests. This overview on sociallymindful D2D correspondences covers the two its specialized issues and applications. We ordered the cutting edge writing dependent on socially-mindful D2D correspondences' specialized issues and applications. In addition, specialized issues and applications are characterized based on the used social highlights, for example, social-ties, social-network, social-trust, and childishness.

\section{REFERENCES}

[1] B. Zhang, Y. Li, D. Jin, P. Hui, and Z. Han, "Social-aware peer discovery for D2D communications underlaying cellular networks," IEEE Transactions on Wireless Communications, vol. 14, no. 5, pp.

[2] 2426-2439, May 2015. [2] K. W. Choi and Z. Han, "Device-to-device discovery for proximitybased service in LTE-Advanced system," IEEE Journal on Selected Areas in Communications, vol. 33, no. 1, pp. 55-66, Jan. 2015.

[3] Tan F. Wang, Y. Li, Z. Wang, and Z. Yang, "Socialcommunity-aware resource allocation for D2D communications underlaying cellular networks," IEEE Transactions on Vehicular Technology, vol. 65, no. 5, pp. 3628-3640, 2016.

[4] X. Lin, Wang, J. G. Andrews, A. Ghosh, and R. Ratasuk, "An overview of $3 \mathrm{GPP}$ device-to-device proximity services," IEEE Communications Magazine, vol. 52, no. 4, pp. 4048, April 2014.

[5] Y. Xiao, D. Niyato, K.-C. Chen, and Z. Han, "Enhance device-to-device communication with social awareness: a belief-based stable marriage game framework," IEEE Wireless Communications, vol. 23, no. 4, pp. 36-44, Aug. 2016.

[6] X. Chen, Mao , B. Proulx, X. Gong, and J. Zhang, "Exploiting social ties for cooperative D2D communications: a mobile social networking case," IEEE/ACM Transactions on Networking, vol. 23, no. 5, pp. 1471-1484, Oct. 2015.

[7] Y. Li, T. Wu, P. Hui, D. Jin, and S. Chen, "Social-aware D2D communications: qualitative insights and quantitative analysis," IEEE Communications Magazine, vol. 52, no. 6, pp. 150-158, June 2014.

[8] N. Y. Asabere, "Towards a viewpoint of context-aware recommender systems (cars) and services," International Journal of Computer Science and Telecommunications, vol. 4, no. 1, pp. 10-29, 2013.

[9] J. Liu, N. Kato, J. Ma, and N. Kadowaki, "Device-to-device communication in LTE-Advanced networks: a survey," IEEE Communications Surveys \& Tutorials, vol. 17, no. 4, pp. 1923-1940, Dec. 2014. 
[10] A. Asadi, Mass , Q. Wang, and V. Mancuso, "A survey on device-to-device communication in cellular networks," IEEE Communications Surveys \& Tutorials, vol. 16, no. 4, pp. 1801-1819, April 2014.

[11] P. Gandotra and R. K. Jha, "Device-to-device communication in cellular networks: A survey," Journal of Network and Computer Applications, June 2016.

[12] S. Singh, N. Saxena, A. Roy, and H. Kim, "A survey on $5 g$ network technologies from social perspective," IETE Technical Review, pp. 1-10, Feb. 2016.

[13] E. Ahmed, I. Yaqoob, A. Gani, M. Imran, and M. Guizani, "Socialaware resource allocation and optimization for D2D communication," IEEE Wireless Communications, 2017.

[14] D. Feng, L. Lu, Y. Yuan-Wu, G. Y. Li, G. Feng, and S. Li, "Device-todevice communications underlaying cellular networks," IEEE Transactions on Communications, vol. 61, no. 8, pp. 3541-3551, Aug. 2013.

[15] H. Tang, Z. Ding, and B. C. Levy, "Enabling D2D communications through neighbor discovery in LTE cellular networks," IEEE Transactions on Signal Processing, vol. 62, no. 19, pp. 5157-5170, Oct. 2014.
[16] K. J. Zou, M. Wang, K. W. Yang, J. Zhang, W. Sheng, Q. Chen, and X. You, "Proximity discovery for device-todevice communications over a cellular network," IEEE Communications Magazine, vol. 52, no. 6, pp. 98-107, June 2014.

[17] C.-H. Yu, K. Doppler, C. B. Ribeiro, and O. Tirkkonen, "Resource sharing optimization for device-to-device communication underlaying cellular networks," IEEE Transactions on Wireless Communications, vol. 10, no. 8, pp. 2752-2763, Aug. 2011.

[18] B. Zhou, H. Hu, S.-Q. Huang, and H.-H. Chen, "Intracluster device to- device relay algorithm with optimal resource utilization," IEEE transactions on vehicular technology, vol. 62, no. 5, pp. 2315-2326, June 2013.

[19] M. Botsov, M. Klügel, W. Kellerer, and P. Fertl, "Location dependent resource allocation for mobile device-todevice communications," in 2014 IEEE Wireless Communications and Networking Conference (WCNC). IEEE, Apr. 2014, pp. 1679-1684.

[20] J. Liu, B. Li, B. Lan, and J. Chang, "A resource reuse scheme of D2D communication underlaying LTE network with intercell interference," Communications and Network, vol. 5, no. 03, p. 187, Sept. 2013. 